\title{
Yeast Dynamically Modify Their Environment to Achieve Better Mating Efficiency
}

\author{
Meng Jin ${ }^{1}$, Beverly Errede ${ }^{1,{ }^{*}}$, Marcelo Behar ${ }^{2}$, Will Mather ${ }^{3}$, Sujata Nayak ${ }^{3}$, Jeff Hasty ${ }^{3}$, \\ Henrik G. DohIman ${ }^{1,4}$, and Timothy C. Elston ${ }^{4,}{ }^{*}$ \\ ${ }^{1}$ Department of Biochemistry and Biophysics, University of North Carolina, Chapel Hill, NC \\ 27599, USA \\ ${ }^{2}$ Department of Chemistry, University of California, San Diego, La Jolla, CA 92093, USA \\ ${ }^{3}$ Division of Biological Sciences, University of California, San Diego, La Jolla, CA 92093, USA \\ ${ }^{4}$ Department of Pharmacology, University of North Carolina, Chapel Hill, NC 27599, USA
}

\begin{abstract}
The maintenance and detection of signaling gradients are critical for proper development and cell migration. In single-cell organisms, gradient detection allows cells to orient toward a distant mating partner or nutrient source. Budding yeast expand their growth toward mating pheromone gradients through a process known as chemotropic growth. MATa cells secrete a-factor pheromone that stimulates chemotropism and mating differentiation in MATa cells and vice versa. Paradoxically, MATa cells secrete Bar1, a protease that degrades a-factor and that attenuates the mating response, yet is also required for efficient mating. We observed that MATa cells avoid each other during chemotropic growth. To explore this behavior, we developed a computational platform to simulate chemotropic growth. Our simulations indicated that the release of Bar1 enabled individual MATa cells to act as a-factor sinks. The simulations suggested that the resultant local reshaping of pheromone concentration created gradients that were directed away from neighboring MATa cells (self-avoidance) and that were increasingly amplified toward partners of the opposite sex during elongation. The behavior of Bar1-deficient cells in gradient chambers and mating assays supported these predictions from the simulations. Thus, budding yeast dynamically remodel their environment to ensure productive responses to an external stimulus and avoid nonproductive cell-cell interactions.
\end{abstract}

\section{INTRODUCTION}

In the yeast Saccharomyces cerevisiae, haploid MATa and MATa cells secrete cell typespecific pheromones (a-factor and a-factor, respectively) that promote cell fusion and the formation of a $M A T \mathbf{a} / M A T a$ diploid. Pheromone stimulation leads to a well-defined series of events required for mating, including mitogen-activated protein kinase (MAPK) phosphorylation, changes in gene transcription, and morphological changes. In response to high concentrations of pheromone, cells arrest in $\mathrm{G}_{1}$ and form the characteristic "shmoo"

\footnotetext{
Copyright 2008 by the American Association for the Advancement of Science; all rights reserved.

*To whom correspondence should be addressed. errede@email.unc.edu (B.E.); telston@ med.unc.edu (T.C.E.).

Author contributions: M.J. and B.E. devised the mating assays and performed the experiments. M.J., M.B., and T.C.E. devised the computational investigations. M.J. and M.B. developed the computational platform for simulating chemotropic growth and M.J. performed the simulations. S.N., W.M., and J.H. devised the microfluidic experiments, and S.N. and W.M. performed the gradient experiments. M.J., B.E., H.G.D., and T.C.E. wrote the manuscript.

Simulation availability: http://www.med.unc.edu/pharm/elstonlab/ResearchSignalTransduction.html.
} 
morphology. However, at intermediate pheromone concentrations, cells undergo chemotropic growth in which they elongate in the direction of increasing pheromone concentrations (1-5).

A property that distinguishes MATa from MATa cells is that MATa cells secrete the protease Bar1 that degrades a-factor $(6,7)$, whereas $M A T a$ cells lack an a-factor-specific protease. It is widely thought that Bar1 serves to "desensitize" the pheromone pathway and restore normal cell division if mating is unsuccessful (6-8). However, Bar1 also has a positive role in mating, because barl null mutant cells are inefficient in finding a mating partner when presented with a mixture of pheromone-producing and non-producing MATa cells (9). Using an analogy to electrostatics, Barkai et al. postulated that a homogenous concentration of Bar1 limits the diffusion range of a-factor, thereby creating local pheromone gradients that are better aligned with the direction of the nearest MATa cell (10). Alby et al. observed that a secreted protease helps to prevent rare same-sex mating in Candida albicans (11). Here, we combine experimental and computational approaches to explore how Bar1 promotes accurate gradient detection and proper mating behavior. In particular, we showed that Bar1 allowed cells of the same mating type to avoid one another, thereby minimizing unproductive encounters, and dynamically magnified pheromone gradients in the direction of the opposite mating type.

\section{RESULTS}

\section{Bar1 provides a mechanism for self-avoidance}

Experiments performed in a microfluidic gradient chamber showed that MATa cells tended to avoid each other as they elongated (Fig. 1A, left panel, and movie S1A). Self-avoidance could contribute to increased mating efficiency by reducing nonproductive encounters with cells of the same mating type. We postulated that self-avoidance behavior might depend on Bar1, which has been shown to have a positive role with respect to mating partner selection (9).

To investigate whether Bar1 is sufficient to explain the self-avoidance behavior, we developed a computational platform for simulating chemotropic growth (Fig. 1, B and C), which is briefly described here (see Materials and Methods for details). The microfluidic chamber is taken to be a square domain, and MATa cells within the chamber are initially represented as circles (Fig. 1B). Bar1 is released from MATa cells and degrades a-factor in the surrounding fluid. To simulate the pheromone gradient inside the microfluidic chamber, we held the pheromone concentration at the right edge of the computational domain at 100 $\mathrm{nM}$ and set the pheromone concentration at the left boundary at zero. The concentrations of Bar1 and pheromone are governed by Eqs. 1 and 2, respectively, in Materials and Methods. These equations are run to steady state to determine the initial concentration profiles.

The $K_{\mathrm{d}}$ for the pheromone receptor Ste 2 has been reported to be $\sim 5 \mathrm{nM}$ (12). Therefore, we assumed that a local pheromone concentration of at least $1 \mathrm{nM}$ was required to initiate a response and that gradient detection was possible for local pheromone concentrations ranging from 1 to $50 \mathrm{nM}$. We modeled cell elongation by inserting successive growth segments into the cell (Fig. 1C) and recalculated the concentration profiles of Bar1 and pheromone after each elongation step. The computed relative a-factor gradient determined the initial direction of growth. The relative gradient is the difference in a-factor concentration across the cell divided by the average concentration over the same region and is therefore a dimensionless quantity. On the basis of measurements made in our microfluidics chamber, we set a relative gradient of 0.025 as the threshold for detectable pheromone gradients (5). For steeper gradients, cells elongate in the direction of the pheromone gradient. For gradients below the threshold, elongation occurs in a random 
direction. Once a cell has begun to elongate (hereafter, "grow"), Bar1 release occurs in a polarized fashion from the leading edge and the relative gradient is measured across the leading growth segment (see Materials and Methods). To model pheromone-induced synthesis of Bar1, we assumed that the synthesis rate increases proportionally to the size of the growing cell. If the gradient becomes subthreshold during elongation, then the cell continues to grow in the same direction; otherwise, the cell reorients in the direction of the gradient (Fig. 1C).

Our simulations indicated that Bar1 released locally from MATa cells was sufficient for these cells to avoid one another within the gradient chamber (Fig. 1D and movie S1B). Indeed, the results of our simulations were similar to the behavior observed experimentally (Fig. 1A, left panel). In particular, our simulation reproduced the situation in which two neighboring MATa cells having initiated growth opposite the a-factor gradient turned away from each other as they reoriented toward the gradient (Fig. 1D, left panel). In this scenario, cells lacking Bar1 would be equally likely to turn toward each other as apart from one another.

To confirm a role for Bar1 in self-avoidance, we repeated the gradient experiments with cells that lack $B A R 1$. In contrast to wild-type cells, bar $1 \triangle$ cells elongated parallel to one another and frequently collided (Fig. 1A, right panel, and movie S1C). To quantify the effects of Bar1, we measured the angle between MATa cells that were initially adjacent in the gradient chamber (Fig. 1E). For cells expressing Bar1, the average angle between two neighbor cells $\left(93.9^{\circ} \pm 2.3^{\circ}, n=22\right)$ was larger than that observed for the bar $1 \Delta$ cells $\left(43.4^{\circ}\right.$ $\pm 4.0^{\circ}, n=15$ ). We then measured the angle between elongating cells and the direction of the gradient (taken to be $0^{\circ}$ ). After sufficiently long times ( $350 \mathrm{~min}$ ), essentially the same number of $B A R 1$ and bar1 $1 \triangle$ cells aligned their growth with the gradient (Fig. 1F).

\section{Local release of Bar1 contributes to mating efficiency}

To quantify the contribution of Bar1 to mating preference, we performed mating assays with $M A T$ a cells either expressing or lacking Bar1. In both cases, we mixed a population of $M A T \mathbf{a}$ cells with an equal number of MATa or MATa cells. The mating partners had complementary selectable markers, allowing quantitation of mating frequencies. Bar1 promoted mating between MATa and MATa cells (Fig. 2A), but diminished rare matings between MATa cells (Fig. 2B). (Note the difference in scale of the $y$ axis between Fig. 2, A and B.) We also examined the effects of exogenously added pheromone on mating. In this case, opposite-sex mating efficiency decreased with increasing pheromone concentration whether cells were $B A R 1$ or $\operatorname{bar} 1 \triangle$. The difference between $B A R 1$ and $\operatorname{bar} 1 \triangle$ cells was less pronounced at the highest concentration of pheromone presumably because at this concentration even cells expressing Bar1 are unable to detect pheromone gradients (3). In contrast to opposite-sex mating, added pheromone increased same-sex matings whether cells were $B A R 1$ or bar1 $\triangle$ (Fig. 2B). This positive effect can be understood because same-sex mating is attributed to autocrine signaling $(13,14)$, which is enhanced by exogenous pheromone. Diploid cells from the same-sex mating assay successfully mated with a MATa tester strain and did not sporulate, verifying that these cells did not result from a rare matingtype switch in the population and subsequent opposite-sex mating events (see Materials and Methods for details).

The data presented in Fig. 2, A and B, demonstrate that Bar1 affects the efficiency of both $M A T \mathbf{a} \times M A T a$ and $M A T \mathbf{a} \times M A T \mathbf{a}$ mating. However, these experiments do not distinguish local versus global effects of the protease. For the case of MATa $\times M A T a$ mating, a uniform background of Bar1 could sharpen a-factor gradients (10) and reduce saturating concentrations of $a$-factor to concentrations where gradient sensing is possible. Additionally, a global reduction of a-factor is expected to diminish the frequency of same- 
sex mating, which depends on autocrine signaling. Our simulations suggested that by causing MATa cells to avoid one another, local gradients of Bar1 may also play a role in reducing same-sex mating. Therefore, we tested whether the local release of Bar1 contributed to the efficiency of either MATa $\times M A T a$ or MATa $\times M A T \mathbf{a}$ mating.

Similar to the mating assays described above, the experiments to evaluate local release of Bar1 involved mixtures of "mating partners" with complementing alleles to allow for the selection of diploid fusion products. Additionally, $B A R 1$ cells were included in experiments involving bar1 $\triangle$ mating partners to provide a source of Bar1 equal to that present in mixtures involving $B A R 1$ mating partners, and bar $1 \triangle$ cells were included in experiments with $B A R 1$ mating partners so that the fraction of selectable cells remained the same as in the case of bar $1 \triangle$ mating partners. These added "equalizer cells" ensure comparable conditions across experiments (see Table 3, crosses 9 to 12 in Materials and Methods). Note that equalizer cells in these crosses mate, but the resulting diploid cells cannot grow on the selection plates because they lack complementing markers and, therefore, are not detectable as mating events. This experimental design ensures that any observed difference in the mating efficiency between mating partners (opposite or same sex) could be attributed to local effects of Bar1.

For opposite-sex mating, MATa cells that expressed Bar1 mated more efficiently than bar1 $\triangle M A T$ a cells even when equalizer cells were included in the mixtures (Fig. 2C). For same-sex mating, MATa bar $1 \triangle$ cells mated more efficiently than $B A R 1$ cells even when equalizer cells were included in the mixtures (Fig. 2D). These results demonstrated that the local release of Bar1 favors opposite-sex mating while disfavoring same-sex encounters. We observed a larger absolute effect of Bar1 on opposite-sex matings as compared with samesex matings. This difference is partially attributable to the fact that the mating assays reveal only those encounters that result in the successful formation of diploids. That is, these experiments do not account for same-sex encounters in which mating is prevented by alternate mechanisms.

\section{Self-avoidance provides an efficient search mechanism}

We next performed simulations to investigate other potential mechanisms for how the local release of Bar1 improves MATa $\times$ MATa mating. Because in the mating assays a large number of $M A T \mathbf{a}$ and $M A T \mathrm{~T}$ cells were mixed together, we expect that the initial a-factor concentration experienced by the MATa cells is fairly homogeneous but that fluctuations in cell density will generate local regions containing clusters of MATa or MATa cells. This scenario is similar to situations in the wild when a cluster of MATa cells encounters a cluster of MATa cells. To investigate the role of Bar1 in such a scenario, we performed simulations in which multiple MATa cells are exposed to a homogenous background of afactor. To establish a spatially uniform a-factor concentration in the absence of MATa cells, we held the pheromone concentration at the boundaries of the computational domain fixed at $50 \mathrm{nM}$. These simulations revealed that MATa cells tended to avoid one another as they elongated so that they grew radially outward away from the center of the colony. This behavior allowed them to explore a large area in search of a mating partner (Fig. 3A). In contrast, when cells lacking Bar1 were started in the same initial geometry, they grew in random directions and frequently collided with each other. Consequently, these cells searched a reduced area (Fig. 3C) and would, therefore, encounter fewer potential mating partners.

To verify the results of these simulations, we used the microfluidic chamber to expose both $B A R 1$ and bar1 $\triangle M A T$ a cells to homogeneous pheromone concentrations. We tracked the direction of cell growth by following green fluorescent protein (GFP) fused to Bem1 (Bem1- 
GFP), a protein that marks the leading edge of polarized growth (15). We found clear qualitative agreement between the simulations and the behavior of both the BAR1 (Fig. 3, A and B) and the bar $1 \Delta$ strains (Fig. 3, C and D). We investigated the range of pheromone concentrations over which MATa cells avoid each other during elongated growth. We used an upper limit for the a-factor concentration of $120 \mathrm{nM}$, because in the gradient chamber above this concentration, the cells do not elongate but form well-defined mating projections. We used a lower limit of $50 \mathrm{nM}$, below which the cells do not respond to pheromone. Between these limits $(75,100$, and $120 \mathrm{nM})$, Bar1-positive cells exhibit self-avoidance (Table 1 and movies S2 to S4).

At concentrations compatible with elongated growth in bar $1 \Delta$ cells ( 5 to $30 \mathrm{nM}$ ) the selfavoidance behavior was absent (Table 1 and movie S5). Notably, the bar1 $1 \Delta$ cells exhibited a high frequency of pairs of cells that elongated parallel to one another during growth in the microfluidic chambers. These pairs represent mother and daughter cells that initially polarized toward each other at the former bud site. The direction of polarization of both cells then rotated until growth was no longer occluded. Consistent with the postulated role for Bar1 in self-avoidance, the pairs of bar1 $1 \triangle$ cells continued to grow parallel rather than diverge from each other as seen for $B A R 1$ cells. Of the 55 mother/daughter pairs counted, 45 exhibited parallel growth (31 grew in the same direction, 14 grew in opposite directions). By contrast, in the $B A R 1$ strain, none of 62 mother/daughter pairs exhibited parallel growth. This ability of Bar1 to prevent mother/daughter pairs from growing parallel to each other may be important in preventing competition for the same mating partner.

\section{Bar1 improves mating efficiency by amplifying pheromone gradients as MATa cells elongate}

Our results indicated that spatial heterogeneities in Bar1 concentration played an important role in shaping the local a-factor concentration. To determine how Bar1 improved the efficiency with which opposite mating types located each other, we used our computational platform to investigate several scenarios involving MATa and MATa cells. In the first case, a single MATa cell was presented with two potential mating partners (Fig. 4A, inset) but was placed closer to the MATa cell on the left. In this geometry, both $B A R 1$ and $\operatorname{bar} 1 \triangle$ cells detect the initial pheromone gradient and follow the same trajectory as they elongate. However, the initial gradient experienced by the $B A R 1$ cell was larger than that of the bar $1 \triangle$ cell and sharpened greatly as the MATa cell elongated (Fig. 4A, red curve). In contrast, without Bar1, the gradient remained shallow (Fig. 4A, blue line).

Next, we considered a geometry in which $B A R 1$ and $\operatorname{bar} 1 \triangle$ cells detected a-factor, but without initially perceiving a spatial gradient. We accomplished this initial condition by placing the MATa cell slightly to the left of the midpoint between the two MATa cells. In this case, we expected both cell types to polarize in a random direction. We tested three representative initial growth directions (Fig. 4B, inset). In all cases, a gradient above the detection threshold quickly developed as the cell with Barl grew (Fig. 4B, red lines), enabling this cell to reorient its direction of growth and find the nearest mating partner (Fig. $4 \mathrm{~B}$, inset red arrows). In contrast, the pheromone gradient across the bar $1 \Delta$ cell remained below the detectable limit (Fig. 4B, blue lines), and cell growth proceeded in the original direction of polarization (Fig. 4B, inset, blue arrows).

Our results suggest that Bar1 improves mating efficiency by progressively amplifying the relative pheromone gradient across $M A T \mathbf{a}$ cells, because the $M A T \mathbf{a}$ cell acts like a pheromone sink where pheromone is continuously degraded by Bar1. If we make the simplifying assumptions that MATa cells are perfect sinks (that is, they absorb all a-factor near them) and $M A T \mathrm{a}$ cells are a constant source of pheromone, then the relative a-factor gradient between the two cells increases as the inverse of the distance between them. This 
prediction is in qualitative agreement with the results of our simulations (Fig. 4, A and B). Thus, our investigations suggest that the release of Bar1 by MATa cells reshapes pheromone concentrations to amplify gradients toward appropriate mating partners. An added advantage of Bar1 remaining highly localized around MATa cells is that gradient amplification is achieved without a drastic reduction in the overall concentration of a-factor.

Finally, we investigated a more complex geometry in which a pair of MATa cells are presented with two potential MATa mating partners (Fig. 5). Both MATa cells were positioned closer to the MATa cell on the left. In the absence of Bar1, the pheromone gradients remained subthreshold so there was no directed growth. If the Bar1 concentration was assumed constant and equal to the concentration near the surface of a MATa cell (1.6 $\mathrm{nM}$ ), then both $M A T \mathbf{a}$ cells detected a gradient and approached the MATa cell on the left (Fig. 5B). However, when the MATa cells released Bar1, the top MATa cell grew away from the bottom MATa cell and toward the MATa cell to the right (Fig. 5C). This result held for a range of initial geometries (Fig. 5A). These simulations showed how the local release of Barl can prevent competition for the same mating partner and thereby improve mating efficiency.

\section{DISCUSSION}

Bar1 promotes mating efficiency despite its ability to degrade the mating stimulus (6-9). Previous computational studies have investigated the role of Bar1 in mating. Barkai et al. (10) proposed that Bar1 improves the alignment of the pheromone gradient with the direction of the nearest MATa cell. Andrews et al. (16) extended this work to show that the local release of Bar1 increases the gradient of liganded receptor across a MATa cell. Here, we considered how the a-factor gradient is dynamically modified as yeast undergo chemotropic growth and navigate toward mating partners. Our results indicated that Bar1 sharpens the relative pheromone gradient as MATa cells elongate. An important consequence is that MATa cells can efficiently adjust their direction of elongation if initial growth is not toward a mating partner.

Our investigations also revealed that local accumulation of Bar1 causes a depletion of $a$ factor between adjacent MATa cells, which in turn produces local pheromone gradients that promote self-avoidance. Thus, the continuous reshaping of local pheromone gradients enables MATa cells to efficiently locate a suitable partner while avoiding interactions unlikely to produce a successful mating event. The insights from our simulations were supported by experimental data showing that bar $1 \Delta$ cells did not exhibit avoidance and instead collided and grew parallel to each other.

Although the effects of Bar1 on same-sex matings are significant, they are substantially less than those reported for the distantly related yeast $C$. albicans (11). Under physiological conditions, $C$. albicans exist primarily as a/a diploids and rarely mate. This physiology contrasts with that of $S$. cerevisiae, where opposite-sex mating is efficient and an important part of its life cycle. Same-sex mating in this life cycle would be deleterious. Consequently, multiple mechanisms exist to limit such events. For example, effects of $S S T 2$ and $A S G 7$ counter autocrine signaling, and the lack of interactions between activated receptors of opposite cell types makes cell fusion inefficient $(13,17)$. Thus, Bar1 alone may provide a sufficient impediment to same-sex mating in $C$. albicans, but multiple mechanisms are in place to prevent the detrimental consequences of same-sex mating in $S$. cerevisiae. Distinguishing the relative contributions of each of these mechanisms to prevent same-sex mating remains an open area of investigation. 
Analogous events have been reported in other systems. For instance, the mechanisms that promote directed migration of neutrophils and Dictyostelium discoideum involve secreted enzymes that serve to degrade the initiating signal (18). In $D$. discoideum, secreted adenosine $3^{\prime}, 5^{\prime}$-monophosphate (cAMP) induces cell migration, aggregation, and differentiation. cAMP is in turn degraded by the secreted phosphodiesterase PdsA. In the absence of PdsA, gradient detection and cellular migration are compromised (19). Thus, just as pheromone gradients in yeast are shaped by the secretion of Bar1, cAMP gradients in $D$. discoidium may likewise be shaped by a secreted cyclic nucleotide phosphodiesterase.

In summary, our findings indicate that hormone proteases can serve dual functions: to reduce the signal and to shape concentration gradients for optimal signal detection and responsiveness. More generally, our findings reveal that cells can dynamically remodel their environment to avoid non-productive cell-cell interactions.

\section{MATERIALS AND METHODS}

\section{Framework for computational simulation of chemotropic growth}

We developed a computational platform for studying yeast chemotropic growth. This platform is built on COMSOL with MATLAB (Comsol Inc.). Schematic diagrams of the simulation platform are given in Fig. 1, B and C. (i) The computational domain that contains the cells is taken to be a square. Initially, cells are assumed to have a circular geometry (Fig. 1B). Biochemical species, reactions, boundary conditions, and initial conditions are defined in the relevant domains. (ii) We assume that cell growth occurs on a longer time scale than the chemical reactions and thermal diffusion. Therefore, all chemical species reach their steady state before cell growth occurs. This simplification allows us to separate solving the reaction-diffusion equations from growing the cells. Solving the reaction-diffusion equations produces the spatial profiles for the extracellular pheromone and Barl concentrations. (iii) The program determines the location of the maximum $\left(P_{\max }\right)$ and minimum $\left(P_{\min }\right)$ pheromone concentration around each cell. The relative gradient is then computed as $2\left(P_{\max }\right.$ $\left.-P_{\min }\right) /\left(P_{\max }+P_{\min }\right)$. If the absolute amount of pheromone and the relative gradient are above the threshold, a narrow rectangular segment is inserted between the half circle defined with $P_{\max }$ as its midpoint and the half circle defined with $P_{\text {back }}$ as its midpoint (Fig. 1C). Otherwise, the cell is elongated in a random or user-specified direction. The half circle, which contains $P_{\max }$ and is moved to accommodate the first inserted segment $\left(\mathrm{S}_{1}\right)$, is defined as the leading edge. The other half circle is taken to be the back of the cell and remains fixed throughout the simulation. (iv) After cell elongation, the program recomputes the steady-state profiles of the reaction-diffusion equations. (v) In later growth steps, only the pheromone concentration across the leading segment is considered, because this is where growth occurs. For this case, the relative gradient is computed as $2\left(P_{\max }-P_{\min }\right) /\left(P_{\max }+\right.$ $\left.P_{\min }\right)$, where $P_{\max }$ and $P_{\min }$ are the maximum and the minimum pheromone concentration, respectively, over the leading segment. If the relative gradient is above the threshold, a new segment $\left(\mathrm{S}_{n+1}\right)$ is inserted between the leading edge and the previous segment and rotated by an angle formed by the previous direction of growth and $P_{\max }$ (Fig. 1C). Otherwise, the new segment is inserted in the same direction as the previous one. These last two steps are repeated until all cells grow in length to three times the original diameter or two cells collide with each other.

\section{Chamber simulations}

For the gradient simulations (Fig. 1B), the concentration of pheromone on the left and right edges of the chamber domain is fixed at 0 and $100 \mathrm{nM}$, respectively, which creates a linear gradient across the chamber domain. In this case, no flux boundary conditions are used along the top and bottom edges of the chamber. For the chamber simulations with a constant 
pheromone background (Fig. 3), the pheromone concentration is fixed at all boundaries of the computational domain. The boundary conditions for Bar1 are absorbing at the four edges (the concentration is taken to be zero at these boundaries). The pheromone and Bar1 concentrations are computed from the following equations:

$$
\begin{aligned}
& \frac{\partial[\mathrm{Bar} 1]}{\partial t}=D_{\mathrm{bar} 1} \Delta[\mathrm{Bar} 1]+s_{\mathrm{b}}(x, y)-d_{\mathrm{b}}[\mathrm{Bar} 1] \\
& \frac{\partial[\mathrm{Ph}]}{\partial t}=D_{\mathrm{ph}} \Delta[\mathrm{Ph}]-k_{\mathrm{b}}[\mathrm{Bar} 1][\mathrm{Ph}]-d_{\mathrm{ph}}[\mathrm{Ph}]
\end{aligned}
$$

where $D_{\mathrm{bar} 1}$ and $D_{\mathrm{ph}}$ are the diffusion coefficients for Bar1 and pheromone, respectively; $s_{\mathrm{b}}(x, y)$ is the secretion of Bar1 from MATa cells; $d_{\mathrm{b}}$ and $d_{\mathrm{ph}}$ are the rate constants for degradation of Bar1 and pheromone, respectively; and $k_{\mathrm{b}}$ is the rate constant for Bar1mediated degradation of pheromone. Initially, MATa cells are assumed to release Bar1 at constant rate uniformly across the entire cell. Once the cells begin to elongate, Bar1 is released in a polarized fashion exclusively from the leading growth segment. The assumption of polarized release of Bar1 during chemotropic growth is not necessary to reproduce the qualitative features of the model. Pheromone-induced Bar1 production is simulated by increasing the flux out of the leading segment in proportion to the size of the cell.

\section{Simulations with MATa and MATa cells}

For simulations involving MATa and MATa cells (Figs. 4 and 5), MATa cells are assumed to produce a-factor uniformly across the cell surface. Bar1 is released from MATa cells. The equation for Bar1 concentrations is the same as Eq. 1, and that for pheromone is now given by

$$
\frac{\partial[\mathrm{Ph}]}{\partial t}=D_{\mathrm{Ph}} \Delta[\mathrm{Ph}]+s_{\mathrm{ph}}(x, y)-k_{\mathrm{b}}[\mathrm{Bar} 1][\mathrm{Ph}]-d_{\mathrm{ph}}[\mathrm{Ph}]
$$

where $s_{\mathrm{ph}}(x, y)$ models the uniform release of a-factor $(75 \mathrm{nM} / \mathrm{s})$ from MATa cells. The growth direction is determined using the relative gradient as described above. No flux boundary conditions (that is, no concentration is lost at the boundaries) are used for a-factor and Bar1.

\section{Estimation of model parameters}

There are seven model parameters: the synthesis and degradation rates of Bar1 and pheromone $\left(s_{\mathrm{Bar} 1}, d_{\mathrm{Bar} 1}, s_{\mathrm{ph}}, d_{\mathrm{ph}}\right)$, the degradation of pheromone by Bar1 $\left(k_{\mathrm{Bar} 1}\right)$, and the diffusion of pheromone and Bar1 $\left(D_{\mathrm{ph}}, D_{\mathrm{Bar} 1}\right)$. We estimated the synthesis, degradation, and diffusion rates of pheromone on the basis of the molecules' size and the generation of a gradient that is $5 \mathrm{nM}$ at the surface of a MATa cell and drops to $20 \%$ of this value $100 \mu \mathrm{m}$ away from the $M A T$ a cell.

We assume that spatial gradients of pheromone and Bar1 exist in only two dimensions ( $x$ and $y$ ). This assumption is valid for the microfluidic chamber, which has a height of $h=5$ $\mu \mathrm{m}$, and for the mating assays, which take place on an agar surface. The pheromone concentration $C_{\mathrm{p}}$ is measured in units of nanomolar (nM). In the absence of other cells, the steady-state profile for $C_{\mathrm{p}}$ around a $M A T \mathrm{Ta}$ cell is described by the following equation: 


$$
\left\{\begin{array}{c}
D_{\mathrm{P}} \nabla^{2} C_{\mathrm{P}}(r)-d_{\mathrm{P}} C_{\mathrm{P}}(r)=0 \\
\left.D_{\mathrm{P}} \frac{\partial C_{\mathrm{P}}(r)}{\partial r}\right|_{r=r_{0}}=-j_{\mathrm{P}} \\
C_{\mathrm{P}}(\infty)=0
\end{array}\right.
$$

where $\nabla^{2} C_{\mathrm{P}}(r)=\frac{\partial^{2} C_{\mathrm{P}}(r)}{\partial r^{2}}+\frac{1}{r} \frac{\partial C_{\mathrm{P}}(r)}{\partial r}$ is the Laplace operator in polar coordinates, $d_{\mathrm{P}}$ is the pheromone degradation rate, $D_{\mathrm{p}}$ is the diffusion coefficient, and $j_{\mathrm{p}}$ is the pheromone flux density [molecules/(area-s)] at the cell boundary located at $r_{0}$. The flux density $j_{\mathrm{P}}$ is computed as follows. Assume pheromone molecules are synthesized inside the cell at a rate of $s_{\mathrm{ph}}(\mathrm{nM} / \mathrm{s})$ and released uniformly over the surface of the cell. Then, the flux per unit area is the product of synthesis rate and the ratio of the cell volume to surface area $j_{\mathrm{p}}=s_{\mathrm{ph}} r_{0} / 3$.

The steady-state solution of Eq. 4 is

$$
C_{\mathrm{P}}(r)=\frac{j_{\mathrm{P}} K_{0}\left(\sqrt{\frac{d_{\mathrm{p}}}{D_{\mathrm{P}}}} r\right)}{\sqrt{d_{\mathrm{P}} D_{\mathrm{P}}} K_{1}\left(\sqrt{\frac{d_{\mathrm{p}}}{D_{\mathrm{P}}}} r_{0}\right)}
$$

where $K_{0}$ and $K_{1}$ are modified Bessel function of the second kind. The diffusion coefficient of pheromone is estimated from its molecular weight as $125 \mu \mathrm{m}^{2} / \mathrm{s}$. On the basis of Eq. 5, we chose the synthesis rate of pheromone to be $75 \mathrm{nM} / \mathrm{s}$ and the degradation rate to be 0.005 $\mathrm{s}^{-1}$. With these values, the pheromone concentration is $5 \mathrm{nM}$ at the surface of a MATa cell and drops to $20 \%$ of this value $100 \mu \mathrm{m}$ away from the source. This synthesis rate requires a MATa cell with 5- $\mu \mathrm{m}$ diameter to produce 3000 pheromone molecules per second.

Bar1 emitted from a MATa cell satisfies an equation analogous to Eq. 4. Because Bar1 is substantially larger than pheromone, we chose its synthesis rate to be $1.5 \mathrm{nM} / \mathrm{s}, 50$ times slower than that of pheromone. Once a MATa cell begins to elongate, we assumed Bar1 is released exclusively from the leading edge. We assumed Bar1 degrades at a rate of $0.05 \mathrm{~s}^{-1}$, which is 10-fold faster compared with pheromone. We take the diffusion coefficient of Bar1 to be $6.25 \mu \mathrm{m}^{2} / \mathrm{s}$, which is 20 times slower than pheromone diffusion coefficient. With these values, the concentration of Bar1 in unit volume at the surface of the cell is $0.85 \mathrm{nM}$ and drops to $20 \%$ of its value at a distance of $13 \mu \mathrm{m}$. The rationale for these choices is given next.

\section{Parameter studies for self-avoidance of adjacent MATa cells}

Among the seven free parameters, we systematically varied $s_{\mathrm{Bar} 1}, d_{\mathrm{Bar} 1}, s_{\mathrm{ph}}$, and $k_{\mathrm{Bar} 1}$ and quantified their effects on self-avoidance and sharpening of the pheromone gradient.

For MATa cells to show self-avoidance (an angle of $>20^{\circ}$ between adjacent cells), the model parameters should satisfy two conditions: (i) Bar1-dependent degradation of pheromone $\left(k_{\mathrm{Bar} 1}[\mathrm{Bar} 1]\right)$ needs to be $\sim 2$ orders of magnitude larger than spontaneous pheromone degradation $\left(d_{\mathrm{ph}}\right)$ (Fig. 6A). This ratio can be increased by increasing the Bar1related parameters $s_{\mathrm{Bar} 1}$ and $k_{\mathrm{Bar} 1}$. Decreasing the Bar1 degradation rate $d_{\mathrm{Bar} 1}$ also affects this ratio. However, this parameter increases the width of Bar1's distribution around a $M A T$ a cell, which is in opposition to the second condition. (ii) We required that the distribution of Bar1 is localized around MATa cells (Fig. 6B). If the Bar1 distribution is too broad, then, because of the first condition, the pheromone concentration around multiple MATa cells is too reduced, making the establishment of sharp pheromone gradients difficult 
and the angle between two neighboring MATa cells small. Two ways to restrict the Bar1 distribution are rapid degradation or slow diffusion. An additional mechanism that would ensure that Bar1 remains localized around MATa cells is if a portion of the protease remained trapped in the periplasmic space between the cell wall and the plasma membrane.

\section{Parameter studies for sharpening pheromone gradients}

Using the same geometry of MATa and MATa cells as in Fig. 4B, we investigated how the ability of Bar1 to sharpen pheromone gradients depended on the model parameters $s_{\mathrm{Ph}}$, $d_{\mathrm{Bar} 1}$, and $k_{\mathrm{Bar} 1}$. The synthesis rate of pheromone, $s_{\mathrm{Ph}}$, changed the amount of pheromone around a MATa cell, but did not affect the relative gradient. Similar to improving selfavoidance, increasing the ratio of $k_{\mathrm{Bar} 1}[\mathrm{Bar} 1] / d_{\mathrm{ph}}$ sharpens pheromone gradients (Fig. 6C). One way to increase this ratio is to decrease the Bar1 degradation rate $d_{\mathrm{Bar} 1}$, which broadens the Bar1 distribution and increases the amount of Bar1 in the medium. A uniform background of Bar1 is sufficient to generate large pheromone gradients, because under this condition, the a-factor distribution is proportional to

$$
K_{0}\left(\sqrt{\frac{k_{\mathrm{Bar} 1}[\mathrm{Bar} 1]}{D_{\mathrm{p}}}} r\right)
$$

which asymptotically decreases as $\exp \left[-\left(k_{\mathrm{Bar} 1}[\mathrm{Bar} 1] / D_{\mathrm{p}}\right)^{1 / 2} r\right]$ for large $r(10)$. However, this sharpening of the gradient comes at the cost of a substantial reduction in the pheromone concentration, making it easy for the absolute amount of pheromone to drop below detection. Keeping the Bar1 concentration localized around MATa cells allows a-factor concentrations to remain relatively high, while at the same time providing a mechanism for amplifying the pheromone gradient as MATa cells elongate.

\section{Yeast strains}

A list of strains used in these studies and their complete genotype is provided in Table 2.

\section{Chemotropic growth assays}

The microfluidic device used for chemotropic assays and preparation of cells for imaging was described previously (5). The pheromone concentration ranged from 0 to $100 \mathrm{nM}$ in the cell chamber for differential interference contrast (DIC) and fluorescence imaging of $B A R 1$ cells (BY4741-15; BEM1-GFP::His3MX6) and 0 to $20 \mathrm{nM}$ for bar1 $\triangle$ cells (BY4741-30; bar1 $1:$ HisG-URA3-HisG BEM1-GFP::His3MXG). To quantify self-avoidance for these two strains, we measured the angle between two adjacent MATa cells every $50 \mathrm{~min}$ from the time that cells started to elongate during chemotropic growth. This angle is defined as the angle between the two lines from the growth tip to the contact point of the two cells. The alignment between the direction of growth and the gradient was quantified by the angle between vectors indicating the growth direction and the direction of gradient at $350 \mathrm{~min}$. Microscopy was performed with a Nikon Ti-E inverted microscope equipped with a Photometrics CoolSNAP HQ2 Monochrome camera. Acquisition was performed with MetaMorph (Molecular Devices). Image processing and analysis was done with MATLAB (MathWorks) and ImageJ (http://rsbweb.nih.gov/ij/).

\section{Quantitative mating assays}

$B A R 1$ and bar1 $\triangle$ strains with nonrevertible and complementing nutritional markers were derived from BY4741 and BY4742 (Table 2) and assessed for opposite cell type (MATa $\times$ $M A T \mathbf{a})$ and same cell type (MATa $\times M A T \mathbf{a})$ mating efficiency in the absence or presence 
of exogenous pheromone, using a modification of the procedure described by Sprague (20). In these experiments, mating mixtures (Table 3 , crosses 1 to 4 ) were made with $1 \times 10^{6}$ cells of each mating partner in suspensions $(200 \mu \mathrm{l})$ containing $0,0.75,1.5,3$, or $6 \mu \mathrm{M}$ exogenous mating pheromone (a-factor). Each suspension was pipetted onto a 25 -mm filter (0.45- $\mu \mathrm{m}$ pore size; Millipore Corp.) on the surface of a separate YPD (yeast extract, peptone, and dextrose) plate with the corresponding concentration of mating pheromone. Cells for these mixtures were grown in liquid YPD medium to the early log phase $\left(5 \times 10^{6}\right.$ to $1.5 \times 10^{7}$ cells $\left./ \mathrm{ml}\right)$. After 5 hours $\left(30^{\circ} \mathrm{C}\right)$, the cells were collected from the filters and diluted for plating on selective medium (synthetic dextrose supplemented with histidine, leucine, and uracil) to determine diploids per milliliter, and on nonselective medium (synthetic complete dextrose) to determine total cells per milliliter (diploids and haploids). Reported mating efficiencies are the ratio of diploids to total cells normalized to that for the reference $M A T \mathbf{a} \times M A T a B A R 1$ mixture without pheromone (Table 3, cross 2). Three independent assays were done for each mating mixture at the specified pheromone concentrations.

In experiments to assess the effects of local Bar1, MATa $B A R 1$ or bar1 $1 \triangle$ equalizer cells were included in the mixtures with mating partners that have complementing selectable markers (Table 3, crosses 9 to 12). MATa $\times M A T \mathbf{a} B A R 1$ or bar1 $1 \Delta$ mating mixtures were made with $2.5 \times 10^{5}$ cells of each mating partner and $5 \times 10^{5}$ cells of the MATa bar1 $1 \Delta$ or $B A R 1$ equalizer strain, respectively. Similarly, MATa $\times M A T a$ a $B A R 1$ or bar $1 \triangle$ mating mixtures were made with $2.5 \times 10^{5}$ cells of each mating partner and $2.5 \times 10^{5}$ cells of the $M A T \mathbf{a}$ barl $1 \triangle$ or $B A R 1$ equalizer strain. (Note that MATa BAR1 cells do not produce or secrete Bar1.) Cell cultures and mating mixtures were prepared and plated to quantify mating events as described above. Mating efficiencies for these comparisons (Table 3 , crosses 9 to 12) are the ratio of diploids to total cells normalized to the MATa $\times M A T a$ $B A R 1$ mixture containing bar $1 \triangle$ equalizer cells (Table 3, cross 10). Three independent assays were done for each mating mixture.

The auxotrophic markers in these strains are coding sequence deletions that are nonrevertible. Therefore, only fusion products with complementing nutritional markers (LYS2/lys $2 \Delta_{O}$ met15 $\Delta_{O} M E T 15$ ) grow on selective medium. To confirm this assertion, we included two plating controls in parallel with the quantitative mating assays. First, $2 \times 10^{6}$ cells of the MATa haploid strains used for mating mixtures 1 and 3 (Table 3) were incubated separately on YPD filters and plated on selective medium at the same dilution as for the MATa $\times$ MATa mating mixtures. Second, crosses between opposite cell-type partners with noncomplementing selectable markers (Table 3, crosses 5 to 8) were made in the absence of exogenous pheromone, incubated on YPD filters, and plated on selective medium at the same dilution as for the MATa $\times M A T a$ mating mixtures. No colonies were observed on any of these control plates.

\section{Cell-type verification}

The cell type of rare diploids from MATa $\times$ MATa mating mixtures was tested to discern whether they are products of same or opposite cell type mating. (The latter could result from mating-type switching in rare cells in the population, a process that involves replacing the a or a allele at the $M A T$ locus with sequence from the $H M L a$ or $H M R$ a locus, respectively.) $M A T \mathbf{a} / M A T \mathbf{a}$ diploid cells mate efficiently with MATa but not with MATa haploids to form viable triploid fusion products. By contrast, $M A T \mathbf{a} / M A T \mathrm{~T}$ diploid cells mate with neither. We performed a qualitative mating assay with tester strains KZ8-5C (MATa) and KZ8-1D (MATa) to test isolated colonies from the selective plates to distinguish between $M A T \mathbf{a} / M A T \mathbf{a}$ and MATa/MATa fusion products (19). Isolates from selective plates corresponding to $M A T \mathbf{a} \times M A T \mathbf{a}$ mating mixtures made without or with the specified 
amounts of exogenous pheromone were tested for mating ability (200 BAR1 and 200 bar1 $\triangle$ isolates from two independent sets of experiments). Forty isolates from the BAR1 MATa $\times$ $M A T a$ mating mixture without exogenous pheromone were included for reference. All 400 diploids that were tested from the MATa $\times$ MATa mixtures mated with the MATa tester but not the MATa tester strain, consistent with assignment of the MATa cell type to these fusion products. As expected, none of the 40 MATa/MATa diploids mated with either tester strain. Additionally, 12 isolates each from selective plates of the MATa $\times M A T \mathbf{a}$ and MATa $\times M A T a$ mixtures without exogenous pheromone were tested for sporulation. No spore asci were observed for products from the same-sex mating mixtures, whereas all of those from the opposite mating-type mixtures produced spore asci.

\section{Supplementary Material}

Refer to Web version on PubMed Central for supplementary material.

\section{Acknowledgments}

Funding: This work was supported by NIH grants GM-079271 (M.J. and T.C.E.), GM-084071 (B.E.), GM-073180 (H.G.D.), and GM-079333 (J.H.).

\section{REFERENCES AND NOTES}

1. Paliwal S, Iglesias PA, Campbell K, Hilioti Z, Groisman A, Levchenko A. MAPK-mediated bimodal gene expression and adaptive gradient sensing in yeast. Nature. 2007; 446:46-51. [PubMed: 17310144]

2. Erdman S, Snyder M. A filamentous growth response mediated by the yeast mating pathway. Genetics. 2001; 159:919-928. [PubMed: 11729141]

3. Dorer R, Pryciak PM, Hartwell LH. Saccharomyces cerevisiae cells execute a default pathway to select a mate in the absence of pheromone gradients. J Cell Biol. 1995; 131:845-861. [PubMed: 7490289]

4. Segall JE. Polarization of yeast cells in spatial gradients of a mating factor. Proc Natl Acad Sci USA. 1993; 90:8332-8336. [PubMed: 8397402]

5. Hao N, Nayak S, Behar M, Shanks RH, Nagiec MJ, Errede B, Hasty J, Elston TC, Dohlman HG. Regulation of cell signaling dynamics by the protein kinase-scaffold Ste5. Mol Cell. 2008; 30:649656. [PubMed: 18538663]

6. Ciejek E, Thorner J. Recovery of $S$. cerevisiae a cells from G1 arrest by a factor pheromone requires endopeptidase action. Cell. 1979; 18:623-635. [PubMed: 391400]

7. Hicks JB, Herskowitz I. Evidence for a new diffusible element of mating pheromones in yeast. Nature. 1976; 260:246-248. [PubMed: 768777]

8. Chan RK, Otte CA. Physiological characterization of Saccharomyces cerevisiae mutants supersensitive to G1 arrest by a factor and a factor pheromones. Mol Cell Biol. 1982; 2:21-29. [PubMed: 7050666]

9. Jackson CL, Hartwell LH. Courtship in S. cerevisiae: Both cell types choose mating partners by responding to the strongest pheromone signal. Cell. 1990; 63:1039-1051. [PubMed: 2257622]

10. Barkai N, Rose MD, Wingreen NS. Protease helps yeast find mating partners. Nature. 1998; 396:422-423. [PubMed: 9853747]

11. Alby K, Schaefer D, Bennett RJ. Homothallic and heterothallic mating in the opportunistic pathogen. Candida albicans Nature. 2009; 460:890-893.

12. Jenness DD, Burkholder AC, Hartwell LH. Binding of a-factor pheromone to Saccharomyces cerevisiae a cells: Dissociation constant and number of binding sites. Mol Cell Biol. 1986; 6:318320. [PubMed: 3023832]

13. Bender A, Sprague GF Jr. Pheromones and pheromone receptors are the primary determinants of mating specificity in the yeast. Saccharomyces cerevisiae Genetics. 1989; 121:463-476. 
14. Whiteway M, Hougan L, Thomas DY. Expression of $M F a 1$ in MATa cells super-sensitive to afactor leads to self-arrest. Mol Gen Genet. 1988; 214:85-88. [PubMed: 3067083]

15. Irazoqui JE, Gladfelter AS, Lew DJ. Scaffold-mediated symmetry breaking by Cdc42p. Nat Cell Biol. 2003; 5:1062-1070. [PubMed: 14625559]

16. Andrews SS, Addy NJ, Brent R, Arkin AP. Detailed simulations of cell biology with Smoldyn 2.1. PLoS Comput Biol. 2010; 6:e1000705. [PubMed: 20300644]

17. Shi C, Kaminskyj S, Caldwell S, Loewen MC. A role for a complex between activated G proteincoupled receptors in yeast cellular mating. Proc Natl Acad Sci USA. 2007; 104:5395-5400. [PubMed: 17369365]

18. Van Haastert PJ, Devreotes PN. Chemotaxis: Signalling the way forward. Nat Rev Mol Cell Biol. 2004; 5:626-634. [PubMed: 15366706]

19. Garcia GL, Rericha EC, Heger CD, Goldsmith PK, Parent CA. The group migration of Dictyostelium cells is regulated by extracellular chemoattractant degradation. Mol Biol Cell. 2009; 20:3295-3304. [PubMed: 19477920]

20. Sprague GF Jr. Assay of yeast mating reaction. Methods Enzymol. 1991; 194:77-93. [PubMed: 2005823] 

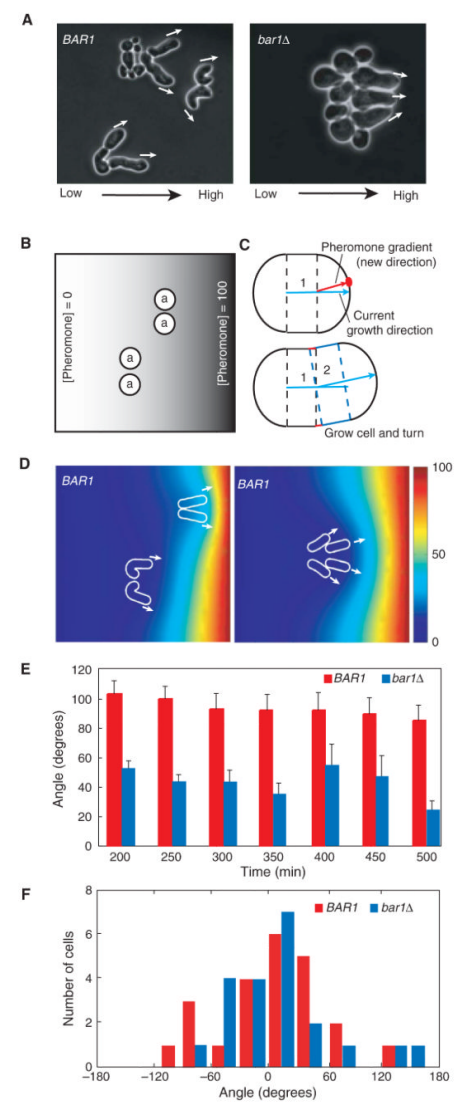

Fig. 1.

Bar1 provides a self-avoidance mechanism. (A) Experiments performed in a microfluidic gradient chamber. BAR1 cells (BY4741-15) show self-avoidance (left panel), whereas bar1 $\triangle$ cells (BY4741-30) tend to grow parallel to one another (right panel). White arrows indicate the direction of growth. Concentration gradient of exogenous pheromone is indicated at the bottom (see movie S1, A and C). (B) Schematic diagram of the initial configuration for simulations of the gradient chamber. The concentrations of pheromone at the left and right sides of the chamber are held at 0 and $100 \mathrm{nM}$, respectively, and the top and bottom sides are assumed to be reflecting. Bar1-positive MATa cells (circles labeled with "a") are placed in the chamber and the Bar1 and pheromone concentrations are computed. (C) An initial growth segment is added between the leading and the back edge of a MATa cell and the pheromone gradient is recomputed (top panel). A second growth segment is inserted and rotated in the direction of the pheromone gradient. The process is repeated. (D) Simulated cell growth demonstrated that MATa cells avoid one another during chemotropic growth in a gradient chamber. White arrows indicate the direction of growth. The color bar at the right shows the color scale for pheromone concentrations (nM) within the computational domains (see movie S1B). (E) Time series for the average angle between adjacent cells in the microfluidic chambers. The average angle is $93.9^{\circ} \pm 2.3^{\circ}(n=22)$ for $B A R 1 M A T \mathbf{a}$ cells (red bars), and $43.4^{\circ} \pm 4.0^{\circ}(n=15)$ for bar $1 \triangle M A T \mathbf{a}$ cells (blue bars). Error bars correspond to $95 \%$ confidence intervals. (F) Histograms for the angle between the direction of the pheromone gradient in the microfluidic chamber and the direction of cell growth at $350 \mathrm{~min}$. 
A

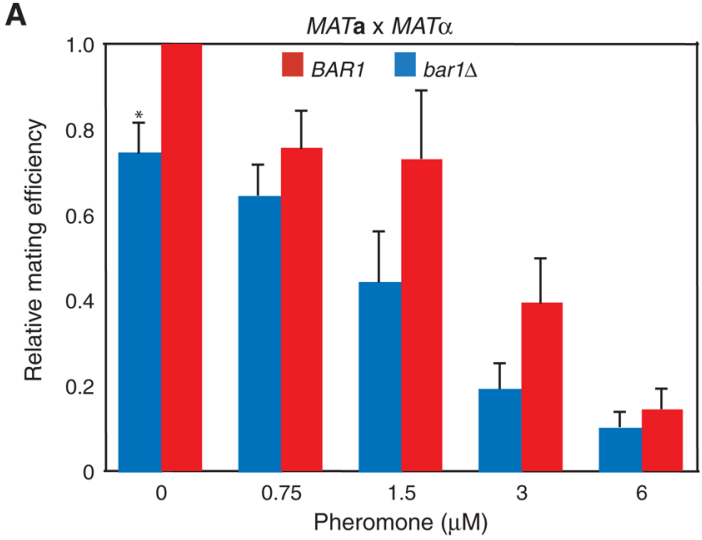

B

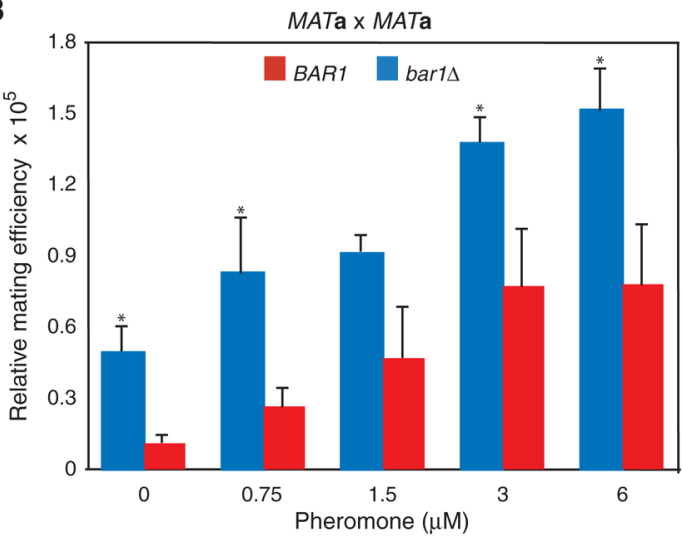

C

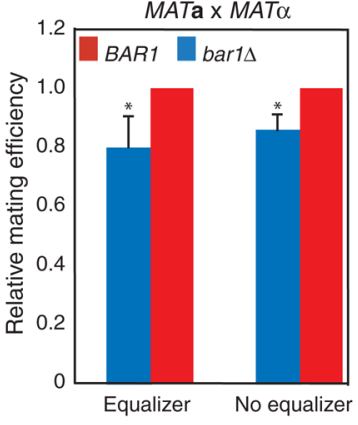

D

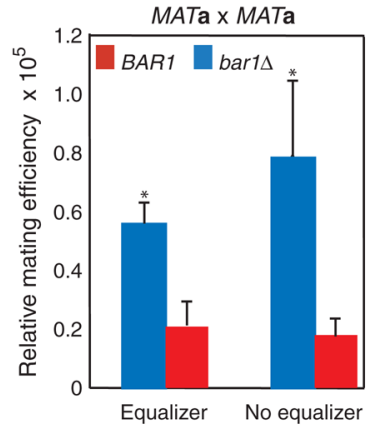

Fig. 2.

Relative mating efficiency for opposite- and same-sex mating. (A) MATa $\times$ MATa mating (Table 3, crosses 2 and 4) at different concentrations of exogenous a-factor. (B) MATa $\times$ MATa mating (Table 3, crosses 1 and 3 ) with the same concentrations of exogenous afactor as in (A). Mating efficiencies in (A) and (B) are relative to that for the MATa $\times$ MATa BAR1 mixture (Table 3, cross 2) with no exogenous pheromone. (C) MATa $\times$ MATa mating without (Table 3, crosses 2 and 4) or with (Table 3, crosses 10 and 12) $M A T \mathbf{a}$ equalizer cells to equalize the amount of Bar1 protease in the mating mixtures. (D) MATa $\times$ MATa mating in the absence (Table 3, crosses 1 and 3) or presence (Table 3, crosses 9 and 11) of MATa equalizer cells to equalize the amount of Bar1 protease in mating mixtures. Mating efficiencies without or with equalizer cells in (C) and (D) are relative to the efficiency of MATa $\times M A T a$ mixtures without (Table 3, cross 2) or with equalizer cells (Table 3, cross 10), respectively. Error bars correspond to 95\% confidence 
intervals. ${ }^{*} P \leq 0.05$ as determined by right-tailed $t$ test; significant difference between $B A R 1$ and $\operatorname{bar} 1 \Delta$ mating parents. 
A

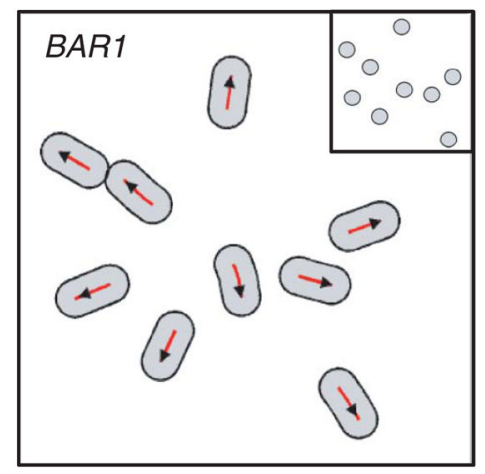

C

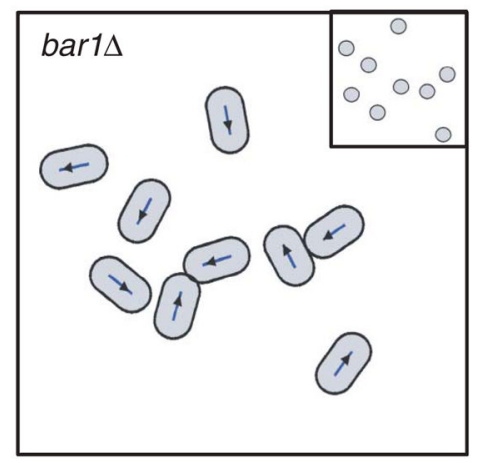

B

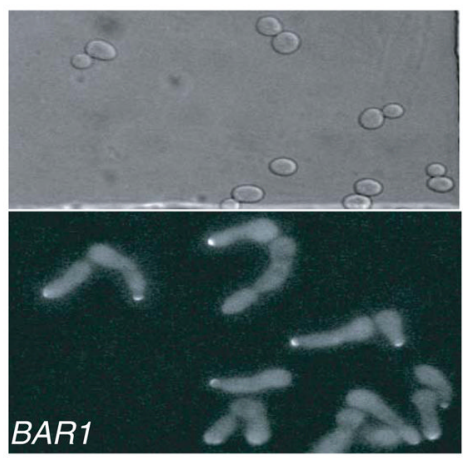

D

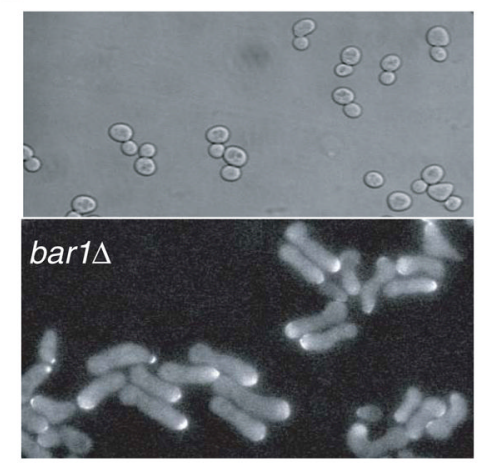

Fig. 3.

Bar1 increases the search area of MATa cells. (A) Simulated growth of $B A R 1$ cells. The localized release of Bar1 causes a-factor gradients to develop so that growth occurs radially outward from the center of the colony. Arrows indicate the direction of growth. (Inset) Initial distribution of MATa cells. (B) Growth of BAR1 cells (BY4741-15) exposed to a constant pheromone background of $100 \mathrm{nM}$ in the microfluidic chamber. The upper panel corresponds to $t=0$ and the lower panel to $t=315 \mathrm{~min}$. (C) Simulated growth of $\operatorname{bar} 1 \Delta$ cells. The $\mathrm{a}$-factor concentration remains constant throughout the computational domain and the cells grow in random directions. (Inset) Initial distribution of MATa cells. (D) Growth of bar1 $\Delta$ cells (BY4741-30) exposed to a constant pheromone background of $10 \mathrm{nM}$ in the microfluidic chamber. The upper panel corresponds to $t=0$ and the lower panel to $t=415$ min. Note the large number of pairs of cells exhibiting parallel growth. In both (B) and (D), the upper panel is a DIC image showing the initial distribution of cells in the chamber, and the lower panel is a fluorescence image showing the chemotropic growth of cells at the indicated times. Bem1-GFP localizes at the leading edge of polarization and shows the direction of cell growth in the chamber. 

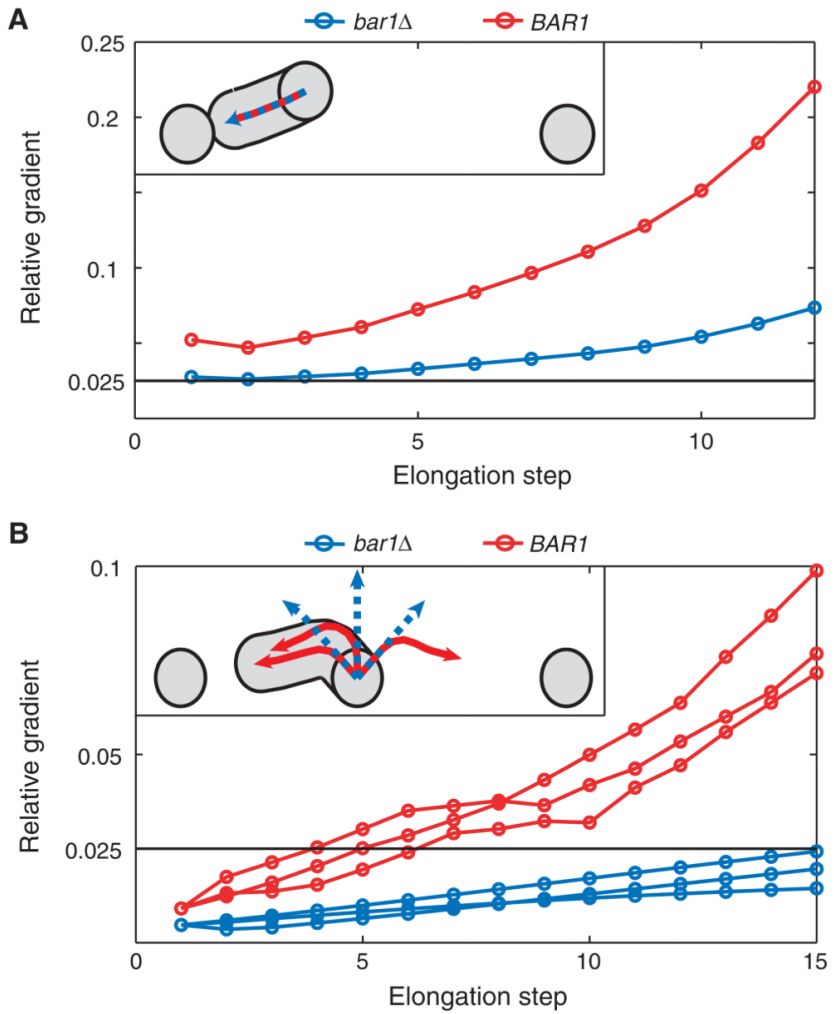

Fig. 4.

Local release of Bar1 amplifies pheromone gradients during chemotropic growth. (A) The relative pheromone gradient (maximum difference in pheromone concentration across the leading growth segment divided by the average concentration over that segment) measured by a $M A T$ a cell producing Bar1 (red curve) or lacking Bar1 (blue curve) as a function of the elongation step. (Inset) The MATa cell is placed close enough to the left MATa cell so that both the $B A R 1$ and the bar1 $\triangle$ cells (indicated by the red and blue lines) can detect a gradient. The arrow indicates the direction of growth. (B) The same simulation as in (A) except that the MATa cell is placed near the midpoint of the two MATa cells, but slightly closer to the left cell. (Inset) Local release of Bar1 allows MATa cells to reorient when the initial relative gradients of $a$-factor are below the 0.025 threshold and initial polarization occurs in a random direction. Red lines in the inset indicate the direction of growth of the $B A R 1$ cells and blue dashed lines indicate the direction of growth of the bar1 $1 \Delta$ cells. 
A

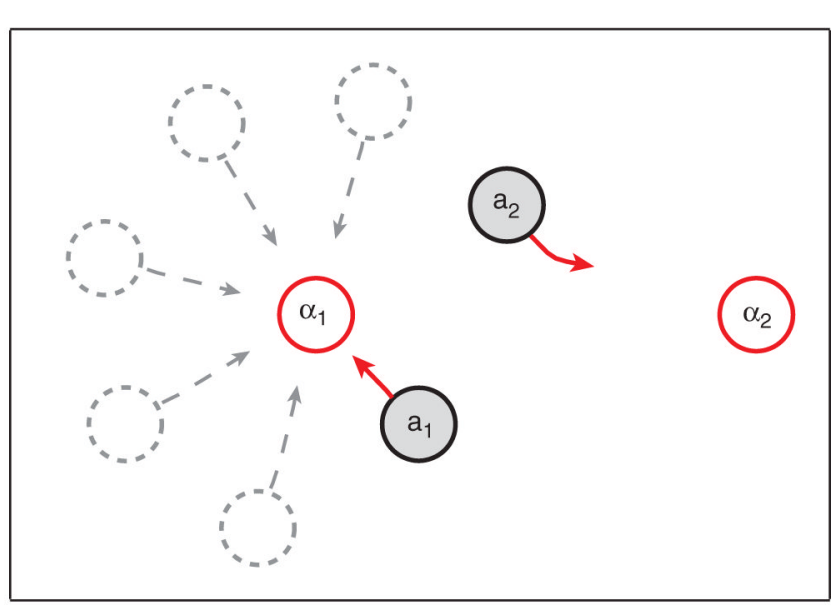

B

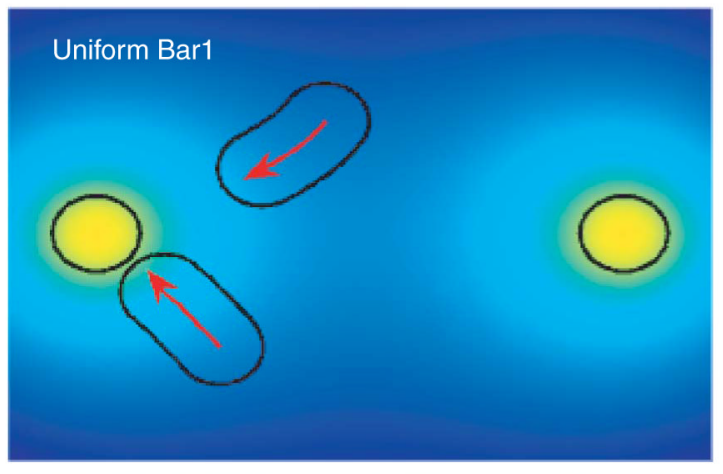

C

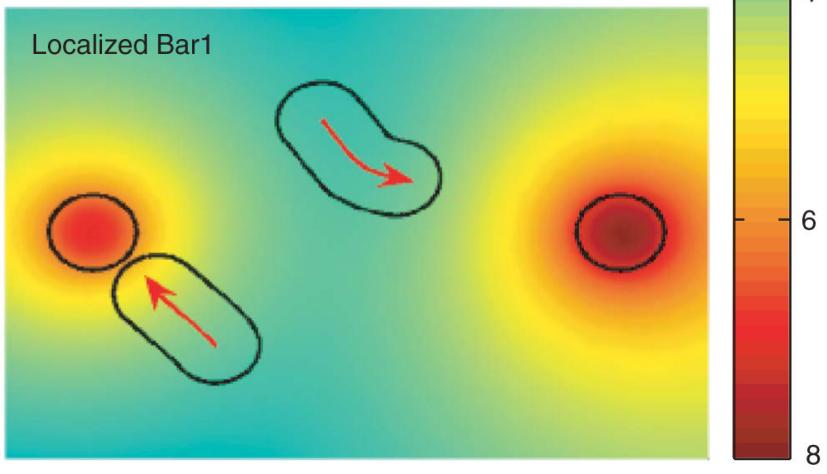

Fig. 5.

Bar1 allows MATa cells to find unique mating partners. (A) Two MATa cells ( $\mathrm{a}_{1}$ and $\mathrm{a}_{2}$ ) are presented with two potential MATa mating partners $\left(\mathrm{a}_{1}\right.$ and $\left.\mathrm{a}_{2}\right)$. The dashed gray circles represent other initial position for $\mathrm{a}_{1}$ that were tested and in each case produced similar results. The red lines with arrowheads represent the direction of growth. (B) In the presence of a uniform background of Bar1, $a_{1}$ and $a_{2}$ compete for $a_{1}$. (C) When $a_{1}$ and $a_{2}$ locally release Bar1, $\mathrm{a}_{2}$ grows away from $\mathrm{a}_{1}$ and toward $\mathrm{a}_{2}$, enabling both cells to find a unique mating partner. The color bar at the right shows the color scale for pheromone concentrations (nM) within the computational domains. 
A

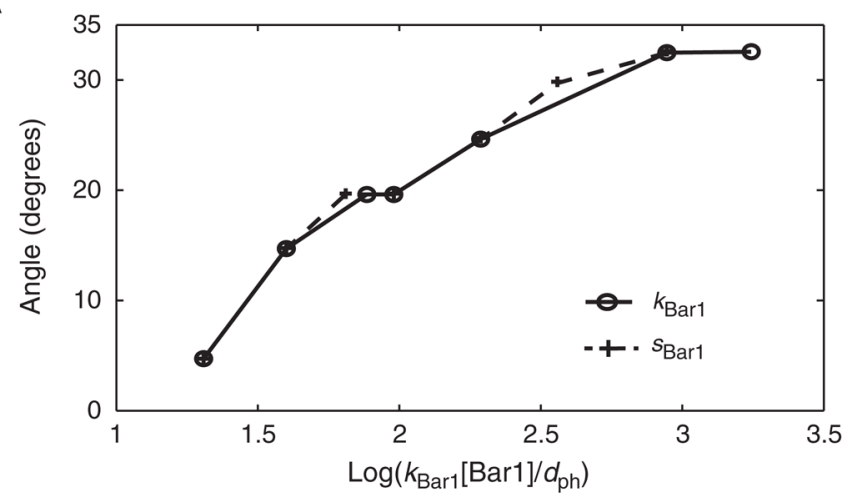

B

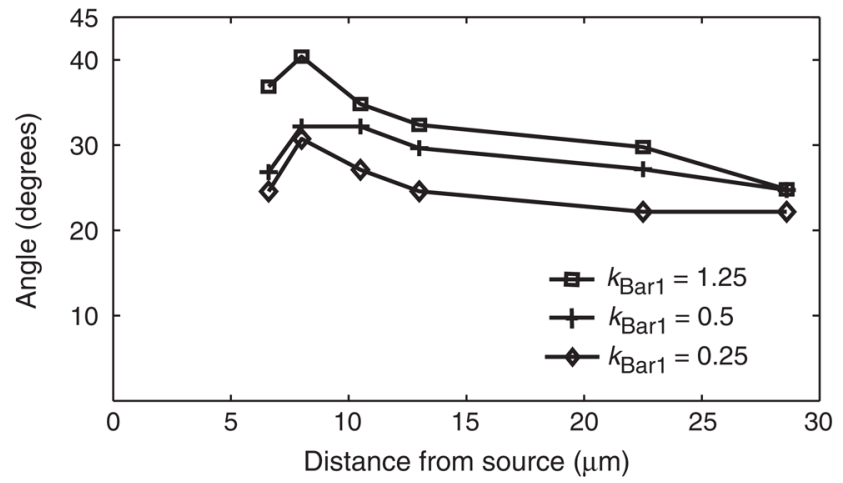

C

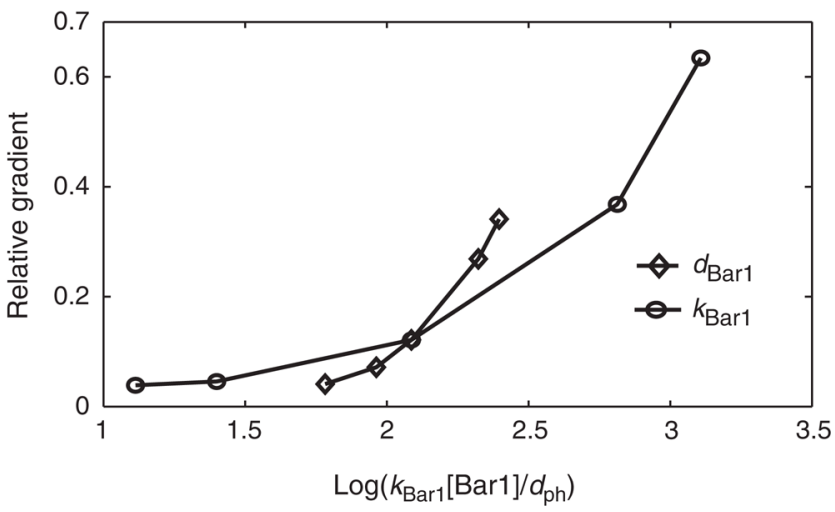

Fig. 6.

Parameter studies. (A) The angle between two adjacent $M A T \mathbf{a}$ cells as a function of $\log \left(k_{\mathrm{Bar} 1}[\mathrm{Bar} 1] / d_{\mathrm{ph}}\right)$. The solid line represents results for varying this ratio by increasing $k_{\mathrm{Bar} 1}$, and the dashed line corresponds to result for changing the ratio by varying the Bar1 synthesis rate, $s_{\mathrm{Bar} 1}$. Both parameters are varied over 2 orders of magnitude. (B) The angle between two adjacent MATa cells as a function of the distance from a MATa cell at which the Bar 1 concentration drops to $20 \%$ of its value. This distance is increased by decreasing $d_{\text {Bar1 }}\left(0.5,0.25,0.1,0.05,0.01\right.$, and $\left.0.005 \mathrm{~s}^{-1}\right)$. The three different curves represent different values of $k_{\mathrm{Bar} 1}$. (C) The relative gradient as a function of $\log \left(k_{\mathrm{Bar} 1}[\mathrm{Bar} 1] / d_{\mathrm{ph}}\right)$. The circles represent results for varying this ratio by changing $k_{\mathrm{Bar} 1}$ and the diamonds are for the case in which this ratio is changed by varying $d_{\text {Barl }}$. 
Table 3

Mating mixtures.

\begin{tabular}{|c|c|c|c|c|}
\hline Cross & Mating partners (equalizer) & Cell type & BAR1 & Selectable marker genotype \\
\hline 1 & BYE007-1B × BYE007-1C & $\mathbf{a} \times \mathbf{a}$ & $B A R 1 \times B A R 1$ & lys $2 \Delta_{O} L Y S 2 \mathrm{MET} 15 / \mathrm{met} 15 \Delta_{0}$ \\
\hline 2 & BYE007-1B × BYE007-2B & $\mathbf{a} \times \mathbf{a}$ & $B A R 1 \times B A R 1$ & lys $2 \Delta_{O} L Y S 2 \mathrm{MET} 15 / \mathrm{met} 15 \Delta_{0}$ \\
\hline 3 & BYE007-2A × BYE007-3A & $\mathbf{a} \times \mathbf{a}$ & $\operatorname{bar} 1 \Delta \times \operatorname{bar} 1 \Delta$ & lys $2 \Delta_{O} L Y S 2 \mathrm{MET} 15 / \mathrm{met} 15 \Delta_{O}$ \\
\hline 4 & BYE007-2A × BYE007-4A & $\mathbf{a} \times \mathbf{a}$ & $\operatorname{bar} 1 \Delta \times \operatorname{bar} 1 \Delta$ & lys $2 \Delta_{o} L Y S 2 \mathrm{MET} 15 / \mathrm{met} 15 \Delta_{O}$ \\
\hline 5 & BYE007-1B × BYE007-6A & $\mathbf{a} \times \mathbf{a}$ & $B A R 1 \times B A R 1$ & lys $2 \Delta_{d} /$ lys $2 \Delta_{O} M E T 15 / M E T 15$ \\
\hline 6 & BYE007-1C × BYE007-2B & $\mathbf{a} \times \mathbf{a}$ & $B A R 1 \times B A R 1$ & $L Y S 2 / L Y S 2$ met $15 \Delta_{d} / m e t 15 \Delta_{0}$ \\
\hline 7 & BYE007-2A × BYE007-14D & $\mathbf{a} \times \mathbf{a}$ & $\operatorname{bar} 1 \Delta \times \operatorname{bar} 1 \Delta$ & lys $2 \Delta_{O} / 1 y s 2 \Delta_{0} M E T 15 / M E T 15$ \\
\hline 8 & BYE007-3A × BYE007-4A & $\mathbf{a} \times \mathbf{a}$ & $\operatorname{bar} 1 \Delta \times \operatorname{bar} 1 \Delta$ & $L Y S 2 / L Y S 2$ met $15 \Delta_{O} /$ met $15 \Delta_{O}$ \\
\hline \multirow[t]{2}{*}{9} & BYE007-1B × BYE007-1C & $\mathbf{a} \times \mathbf{a}$ & $B A R 1 \times B A R 1$ & lys $2 \Delta_{O} / L Y S 2$ MET15/met $15 \Delta_{O}$ \\
\hline & (BYE007-5B) & $\mathbf{a}$ & bar1 $1 \Delta$ & lys $2 \Delta_{0}$ met $15 \Delta_{0}$ \\
\hline \multirow[t]{2}{*}{10} & BYE007-1B $\times$ BYE007-2B & $\mathbf{a} \times \mathbf{a}$ & $B A R 1 \times B A R 1$ & lys $2 \Delta_{0} / L Y S 2$ MET15/met $15 \Delta_{0}$ \\
\hline & (BYE007-5B) & a & bar1 $1 \Delta$ & lys $2 \Delta_{0}$ met $15 \Delta_{0}$ \\
\hline \multirow[t]{2}{*}{11} & BYE007-2A × BYE007-3A & $\mathbf{a} \times \mathbf{a}$ & $\operatorname{bar} 1 \Delta \times \operatorname{bar} 1 \Delta$ & lys $2 \Delta_{O} / L Y S 2$ MET15/met $15 \Delta_{O}$ \\
\hline & (BYE007-6C) & $\mathbf{a}$ & $B A R 1$ & lys $2 \Delta_{0}$ met $15 \Delta_{0}$ \\
\hline \multirow[t]{2}{*}{12} & BYE007-2A × BYE007-4A & $\mathbf{a} \times \mathbf{a}$ & $\operatorname{bar} 1 \Delta \times \operatorname{bar} 1 \Delta$ & lys $2 \Delta_{O} L Y Y S 2$ MET15/met $15 \Delta_{0}$ \\
\hline & (BYE007-6C) & $\mathbf{a}$ & $B A R 1$ & lys $2 \Delta_{O}$ met $15 \Delta_{O}$ \\
\hline
\end{tabular}

